\title{
Class Groups of Quadratic Fields
}

\author{
By Duncan A. Buell
}

\begin{abstract}
The author has computed the class groups of all complex quadratic number fields $Q(\sqrt{-D})$ of discriminant $-D$ for $0<D<4000000$. In so doing, it was found that the first occurrences of rank three in the 3-Sylow subgroup are $D=$ $3321607=$ prime, class group $C(3) \times C(3) \times C(9.7)(C(n)$ a cyclic group of order $n)$, and $D=3640387=421.8647$, class group $C(3) \times C(3) \times C(9.2)$. The author has also found polynomials representing discriminants of 3-rank $\geqslant 2$, and has found 3-rank 3 for $D=6562327=367.17881,8124503,10676983,193816927$, all prime, $390240895=5.11 .7095289$, and $503450951=$ prime. The first five of these were discovered by Diaz y Diaz, using a different method. The author believes, however, that his computation independently establishes the fact that 3321607 and 3640387 are the smallest $D$ with 3-rank 3.

The smallest examples of noncyclic 13-, 17-, and 19-Sylow subgroups have been found, and of groups noncyclic in two odd $p$-Sylow subgroups. $D=119191=$ prime, class group $C(15) \times C(15)$, had been found by A. O. L. Atkin; the next such $D$ is $2075343=3.17 .40693$, class group $C(30) \times C(30)$. Finally, $D=3561799=$ prime has class group $C(21) \times C(63)$, the smallest $D$ noncyclic for 3 and 7 together.
\end{abstract}

Introduction. Throughout this paper, $-D<0$ will denote the discriminant of an imaginary quadratic number field, and "smallest" will refer to $D$, not to $-D$, so that "smallest $D$ " means "largest discriminant."

The author has computed the class groups of all quadratic number fields $Q(\sqrt{-d})$, $d>0$, of discriminant $-D$, for $0<D<4000000$. By a theorem of Gauss, if $D$ has $k$ distinct prime factors, the 2-Sylow subgroup of the class group has rank $k-1$. Apart from this, the groups tend to be cyclic. Even the 2-Sylow subgroup tends to be $k-2$ elementary 2-groups and one large cyclic factor collecting the other powers of two in the class number, so that the 2-Sylow subgroup of the subgroup of squares is cyclic. In computing the 2-Sylow subgroup, then, we actually computed that subgroup of the subgroup of squares, and shall, by abuse of language, call this the 2-Sylow subgroup, calling the group cyclic if the subgroup of squares is. The subgroup of squares is, in the terminology of Gauss, the principal genus, and a discriminant for which the principal genus is noncyclic is called irregular. Thus, what we call a discriminant with a noncyclic 2-Sylow subgroup is a discriminant which Gauss would call irregular.

Statistics were kept on the frequency of occurrence of noncyclic groups, and of the noncyclic $p$-Sylow subgroups for $p=2,3,5,7$. Special listings were also made of the noncyclic $p$-Sylow subgroups for $p \geqslant 11$, of the $p$-Sylow subgroups $C\left(p^{a}\right) \times C\left(p^{b}\right)$ with $a$ and $b \geqslant 2$, and of the class groups noncyclic in more than one $p$-Sylow subgroup. We note here that $95.74 \%$ of the class groups turned out to be cyclic. The

Received June 3, 1975; revised October 14, 1975.

AMS (MOS) subject classifications (1970). Primary 12-04, $12 \mathrm{~A} 25$.

Copyright $\odot$ 1976, American Mathematical Society 
method of computation is outlined in Appendix A; the results are listed and discussed in Appendix B.

In the process of computation, we found that $D=3321607=$ prime has class group $C(3) \times C(3) \times C(9.7)$, and $D=3640387=421.8647$ has class group $C(3) \times$ $C(3) \times C(9.2)$. These are the smallest $D$ for which the class group has $p$-rank greater than two, for $p \geqslant 3$. That an infinity of discriminants with 3-rank 3 exists has been proved by Craig [1] , and numerous examples were given by Shanks and his collaborators [2]-[5], the smallest $D$ being 63199139 .

We developed a method for obtaining quartic polynomials representing, for all squarefree negative integer values within certain bounds, discriminants with 3-rank $\geqslant 2$. With this method, we found six more discriminants with 3-rank 3. The method and these immediate results are detailed in Section 1.

Subsequent to our investigation, we learned that Diaz y Diaz [6], [6a] had made, by an entirely different method, an extensive search for discriminants with 3-rank $\geqslant 3$, and had found, in addition to our five smallest $D$, ninety-four others smaller than 63199139. We feel, however, that our computation is the first complete verification of the fact that 3321607 and 3640387 are the smallest $D$ with 3-rank 3.

In Section 2, we investigate an identical relation between forms obtained by the method of Section 1, and obtain a connection between the group composition of forms of discriminant $-D$ and the group law of the elliptic curve $Y^{2}=4 X^{3}-D$. Finally, in Section 3, we consider problems and conjectures of our method.

All of the computations were made on the IBM 370/158 computer at the University of Illinois at Chicago Circle, Chicago, Illinois; we thank the University for making the computer facilities available.

1. Let $D$ be a positive squarefree integer. The solutions in integers of the Diophantine equation

$$
4 a^{3}=b^{2}+c^{2} D, \quad 0<a<\sqrt{D / 3}, 0<b,(b, c) \leqslant 2,
$$

correspond to ideals $a=(a,(b+c \sqrt{-D}) / 2)$ in the ring of integers of $Q(\sqrt{-D})$ whose cube is principal: $a^{3}=((b+c \sqrt{-D}) / 2)$ [4]. More simply, if $(a, b)=1$, there is a quadratic form $\left(a, b, a^{2}\right)$ of discriminant $-c^{2} D$ whose cube is principal:

$$
\left(a, b, a^{2}\right)^{2} \sim\left(a^{2}, b, a\right) \sim\left(a,-b, a^{2}\right) \sim\left(a, b, a^{2}\right)^{-1} .
$$

Let us assume $c=1$ in (1), and drop the restriction on the size of $a$, and call this Eq. (1a). We seek to produce discriminants $-D$ with a large 3-rank in the class group by obtaining parametric representations

$$
-D=\Delta(x)=S_{1}^{2}-4 C_{1}^{3}=S_{2}^{2}-4 C_{2}^{3}=S_{3}^{2}-4 C_{3}^{3}
$$

where the $S_{i}$ and $C_{i}$ are polynomials in $x$ :

$$
S_{i}(x)=x^{2}+a_{i} x+b_{i}, \quad C_{i}(x)=c_{i} x+d_{i}
$$

Then (2) implies

$$
\left(S_{i}+S_{j}\right)\left(S_{i}-S_{j}\right)=4\left(C_{i}-C_{j}\right)\left(C_{i}^{2}+C_{i} C_{j}+C_{j}^{2}\right), \quad 1 \leqslant i, j \leqslant 3, i<j .
$$


If we insist that the linear term on the right divides the linear term on the left, that is,

(3) $S_{1}-S_{2}=K\left(C_{1}-C_{2}\right), \quad S_{1}-S_{3}=K\left(C_{1}-C_{3}\right), \quad S_{2}-S_{3}=K\left(C_{2}-C_{3}\right)$,

for $K$ an integer, we obtain the equations:

$$
\begin{aligned}
b_{1}^{2}-4 d_{1}^{3} & =b_{2}^{2}-4 d_{2}^{3}, \quad\left(b_{1}-b_{2}\right) /\left(d_{1}-d_{2}\right)=K, \quad\left(a_{1}-a_{2}\right) /\left(c_{1}-c_{2}\right)=K, \\
a_{1} & =(K / 2)\left(c_{1}-c_{2}\right)+(2 / K)\left(d_{2}\left(2 c_{2}+c_{1}\right)+d_{1}\left(2 c_{1}+c_{2}\right)\right) \\
2 K & =\left(2 c_{1}+c_{2}\right)^{2}+3 c_{2}^{2}
\end{aligned}
$$

and two other sets of equations from the latter two of Eqs. (3). Considering the last equation in each set, we get, by the theory of automorphs of the form $(1,1,1)$, that $c_{1}+c_{2}+c_{3}=0$. Using this, we can reduce the relations among the coefficients to the following:

$$
\begin{gathered}
b_{1}^{2}-4 d_{1}^{3}=b_{2}^{2}-4 d_{2}^{3}=b_{3}^{2}-4 d_{3}^{3}, \\
\left(b_{1}-b_{2}\right) /\left(d_{1}-d_{2}\right)=\left(b_{1}-b_{3}\right) /\left(d_{1}-\lambda\right)=\left(b_{2}-b_{3}\right) /\left(d_{2}-d_{3}\right)=K, \\
2 K=(2 u+v)^{2}+3 v^{2}, \quad\left(c_{1}, c_{2}, c_{3}\right) \text { some permu. } \text { on of }(u, v,-u-v), \\
a_{1}=c_{1} K+(2 / K)\left(c_{1} d_{1}+c_{2} d_{2}+c_{3} d_{3}\right)=c_{1} K+M, \\
a_{2}=c_{2} K+M, \quad a_{3}=c_{3} K+M .
\end{gathered}
$$

Thus, if we assume $b_{1}, b_{2}, b_{3}, d_{1}, d_{2}, d_{3}, K, u$, and $v$ satisfy the first three of Eqs. (5), we obtain, except in certain special cases, six distinct polynomials $\Delta(x)$ for each solution $(u, v,-u-v)$, corresponding to the two 3-cycles of the solution. (If $K=6 u^{2}$ we have $u=v$, and if $K=2 u^{2}$, we have $u=-v$. In the former instance only one cycle results; in the latter, only one cycle up to a change of sign, which, as we shall note, does not affect the polynomials obtained.) It appears to be the case, though we have not yet proved it, that exactly one of the two 3-cycles yields integers $a_{i}$, while the other yields only rational solutions in general. In the special cases, the $a_{i}$ appear to be integral. Since the triple $\left(c_{1}, c_{2}, c_{3}\right)$ is determined only up to a sign change, we choose as a convention that the largest of the $\left|c_{i}\right|$ should be chosen negative, noting that $\Delta\left(c_{1}, c_{2}, c_{3}, x\right)=\Delta\left(-c_{1},-c_{2},-c_{3},-x\right)$.

We show now that only the first two of Eqs. (5) are independent; for all $K$ such that the first two equations are solvable, $2 K=u^{2}+3 v^{2}$ has integer solutions $u$ and $v$. The first equation can be partly rewritten

$$
\left(b_{1}+b_{2}\right)\left(b_{1}-b_{2}\right)=\left(d_{1}-d_{2}\right)\left(\left(2 d_{2}+d_{1}\right)^{2}+3 d_{1}^{2}\right) .
$$

Dividing by $4\left(d_{1}-d_{2}\right)$, we obtain

$$
\left(\left(b_{1}+b_{2}\right) / 2\right)(K / 2)=\left(\left(2 d_{2}+d_{1}\right)^{2}+3 d_{1}^{2}\right) / 4 \text {. }
$$

The left-hand side is thus the norm of an integer in $Q(\sqrt{-3})$. The only reason, then, 
that $2 K=u^{2}+3 v^{2}$ might not be solvable would be that $p \mid(K / 2)$ and $p \mid\left(\left(b_{1}+b_{2}\right) / 2\right)$, for $p$ a prime which is $\equiv 2$ modulo 3 . But then we must have $p \mid d_{1}$ and $p \mid\left(2 d_{2}+d_{1}\right)$; there are no primitive representations $p^{2}=u^{2}+3 v^{2}$ for such $p$. That $p \mid(K / 2)$ and $\left(\left(b_{1}+b_{2}\right) / 2\right)$, however, implies that $p \mid b_{1}$ and $p \mid b_{2}$, so that $p^{2} \mid-D=b_{1}^{2}-4 d_{1}^{3}$. Thus, if $D$ is squarefree, $2 K=u^{2}+3 v^{2}$ has integer solutions $u$ and $v$.

We now have a list of discriminants $\Delta(x)$, and we establish conditions for independence of the forms. If $\left(Q, P, Q^{2}\right)$ and $\left(S, R, S^{2}\right)$ lie in the same or in inverse classes, then we can find integers $a, b, c, d$, such that $a d-b c=+1$ and that

$$
4 Q S=(2 Q a+P c)^{2}-c^{2} \Delta(x), \quad 4 Q S=\left(2 S d-R c_{1}\right)^{2}-c^{2} \Delta(x),
$$

where $c_{1}$ equals $c$ if the classes are the same, and $-c$ if they are inverse to each other. If $\Delta(x)<0$ and $4 Q S<-\Delta(x)$, we must choose $c=0$. This forces $S=Q a^{2}$ and $Q=S d^{2}$, which imply $S=Q$. Thus, of the forms $\left(C_{i}, S_{i}, C_{i}^{2}\right)$, if we can satisfy $4 C_{i} C_{j}$ $<-\Delta(x)$ for $\Delta(x)<0$ and $i \neq j$, we are guaranteed that the 3-Sylow subgroup of the class group of $Q(\sqrt{\Delta(x)})$ has rank at least two. (That we cannot guarantee rank three by the existence of the third form will appear in Section 2.)

It remains to be seen that solutions $\left(d_{i}, b_{i}\right), i=1,2,3$, and $K$ exist for the first two of Eqs. (5). The smallest $D$ for which $3 \ln (Q(\sqrt{-D}))$ is $D=23$, and we do indeed find solutions. However, since $\Delta(x)$ is a quartic polynomial, it assumes only finitely many negative integer values, and for these $\Delta(x)$ the discriminants less than zero are much too small to be interesting.

The appearance of $D=3321607$, however, with thirteen pairs of solutions to (1a), provided numerous useful polynomials $\Delta(x)$. From $(94,27),(152,3275),(538$, 24891), $K=56,\left(c_{1}, c_{2}, c_{3}\right)=(4,2,-6)$, we get

$$
\begin{aligned}
\Delta(x) & =\left(x^{2}+133 x+27\right)^{2}-4(4 x+94)^{3}=\left(x^{2}+21 x+3275\right)^{2}-4(2 x+152)^{3} \\
& =\left(x^{2}-427 x+24891\right)^{2}-4(-6 x+538)^{3} .
\end{aligned}
$$

This $\Delta(x)$ is negative for integers $x,-8 \leqslant x \leqslant 75$, and $\Delta(70)=-6562327=$ -367.17881 has class group $C(3) \times C(3) \times C(9.2 .7)$.

From $(152,-3275),(284,9397),(1868,161461), K=96,\left(c_{1}, c_{2}, c_{3}\right)=$ $(4,4,-8)$, we get a $\Delta(x)$ which is negative for integers $x,-1 \leqslant x \leqslant 201 . \Delta(60)=$ $-193816927=-$ prime has class group $C(3) \times C(3) \times C(3.5 .53) . \Delta(108)=$ $-390240895=-5.11 .7095289$ has a class group whose 3-Sylow subgroup is $C(3) \times$ $C(3) \times C(27)$.

From $(128,-2251),(202,5445),(2374,231333), K=104$, with $\left(c_{1}\right.$, $\left.c_{2}, c_{3}\right)=(6,2,-8)$ (which has nonintegral $\left.a_{i}\right)$, we find that $\Delta(169)=-503450951=$ - prime has class group $C(3) \times C(3) \times C(27.103)$.

We now take the constant in $\Delta(x)$ to be -6562327 , and choose $(118,99)$, (248, 7379), (418, 16899), for which $K=56$. If we let $\left(c_{1}, c_{2}, c_{3}\right)=(2,-6,4)$, $\Delta(7)=-8124503=-$ prime has class group $C(3) \times C(3) \times C(9.29)$; if we let $\left(c_{1}, c_{2}\right.$, $\left.c_{3}\right)=(4,2,-6)$, we find that $\Delta(7)=-10676983=-$ prime has class group $C(3) \times$ $C(3) \times C(3.5 .7)$. 
2. The group law on an elliptic curve may be described simply by saying that collinear points sum to zero. That is, if the three (counting multiplicities) points of intersection of the curve with a straight line are $P_{1}, P_{2}$, and $P_{3}$, then $P_{1}+P_{2}+P_{3}=0$.

The composition of binary quadratic forms is considerably more complicated, in part because the elements of the group are not the forms themselves, but the classes of forms equivalent under the transformations of the modular group. One algorithm for composition is as follows:

To compound $(a, b, c)$ with $\left(a^{\prime}, b^{\prime}, c^{\prime}\right)$, both of discriminant $-D$, let $b^{\prime \prime}=$ $\left(b+b^{\prime}\right) / 2, m=\left(a, b^{\prime \prime}\right)$, and $n=\left(a^{\prime}, m\right)$. Solve the equation $a x+b^{\prime \prime} y=m$ for $x$ and $y$, and then the congruence

$$
m z / n \equiv\left(b^{\prime \prime}-b\right) x-c y \quad\left(\bmod a^{\prime} / n\right)
$$

for $z$. If we then let $A=a a^{\prime} / n^{2}, B=b+2 a z / n$, and $C=\left(B^{2}+D\right) / 4 A$, the class of $(A, B, C)$ is that compounded of the classes of $(a, b, c)$ and $\left(a^{\prime}, b^{\prime}, c^{\prime}\right)$. In general, $(A, B, C)$ will not be reduced.

Now, let $D \equiv-1(\bmod 4)$ be a squarefree positive integer, and let $\left(d_{i}, b_{i}\right), i=$ $1,2,3$, be the points of intersection of the elliptic curve $Y^{2}=4 X^{3}-D$ with the line $Y=K X+L$. We assume that $d_{1}, b_{1}, d_{2}, b_{2}$, and $K$ are integers, and let $F_{1}=\left(d_{1}\right.$, $\left.b_{1}, d_{1}^{2}\right)$ and $F_{2}=\left(d_{2}, b_{2}, d_{2}^{2}\right)$ be the corresponding forms. We note, as symmetric functions of the roots, that

$$
K^{2} / 4=d_{1}+d_{2}+d_{3} \text { and } L^{2}+D=4 d_{1} d_{2} d_{3} .
$$

By the first of these, $K$ is even if and only if $d_{3}$ (and hence $b_{3}$ ) is integral. In this case, we have a third form $F_{3}=\left(d_{3}, b_{3}, d_{3}^{2}\right)$.

If $K$ is odd, then $d_{3}=t / 4$ and $b_{3}=u / 4$, for $t$ and $u$ odd integers. We notice $4 t^{3}=(2 u)^{2}+64 D$; barring the restriction on the size of $a$, we have a solution of (1). The ideal which we obtain is $a=(t, u+4 \sqrt{-D})$. Dividing (1) by 4 , we notice that $t \equiv 1(\bmod 8)$, and we rewrite the basis of $a$ as follows:

$$
\begin{aligned}
a & =(t, u+4 \sqrt{-D}, u(1-t) / 4+(1-t) \sqrt{-D})=(t, u+4 \sqrt{-D}, u(1-t) / 4+\sqrt{-D}) \\
& =(t, u(1-t) / 4+\sqrt{-D})=(t, u(1-t) / 4+r t+\sqrt{-D})=(t, U),
\end{aligned}
$$

where $r$ can be any integer. We choose $r$ to be an odd integer such that $t \gamma(u-4 r)$. Then the norm of $U$ is $t s$, where $s$ is prime to $t$, and $t$ is the norm of $a$. Since $t$ is odd, we can choose as basis $t$ and $U / 2: a=(t, U / 2)$. We note that since $t \equiv 1(\bmod$ 8 ), and $r$ is assumed odd, $U$ is odd, and $(U+\sqrt{-D}) / 2$ is an integer in $Q(\sqrt{-D})$. By the correspondence between classes of ideals and classes of forms [7], $a$ induces a form $(t, U, v)$ of discriminant $-D$, and has $t$ and $U / 2$ as an integral basis. We choose an equivalent form for $F_{3}$ :

$$
F_{3}=\left(t, u(1-t) / 4+t, v_{1}\right)
$$

We now state and prove the following

THEOREM. With the above notation,

$$
F_{1} \circ F_{2} \circ F_{3} \sim(1,1,(D+1) / 4)=\text { group identity. }
$$


Thus, the composition of classes of forms coincides with the group law of the elliptic curve.

Proof. We rewrite the first of Eqs. (5):

$$
\left(b_{1}+b_{2}\right) K=4\left(d_{1}^{2}+d_{1} d_{2}+d_{2}^{2}\right) \text {. }
$$

If $p \mid d_{1}$ and $p \mid\left(b_{1}+b_{2}\right)$, for an odd prime $p$, then $p \mid d_{2}$. This, however, implies that $p \mid\left(b_{1}-b_{2}\right)$, hence $p \mid b_{1}$, and $p^{2} \mid D$. Thus, in compounding, $m$ and $n$ are powers of 2 (or are 1). Simple congruences modulo 8 show that $d_{1}$ and $d_{2}$ (and $d_{3}$, if it is integral) are even if and only if $D \equiv-1(\bmod 8)$ and odd if and only if $D \equiv-5(\bmod 8)$. We now distinguish three cases:

(i) $K$ is even. We know that $d_{1}-d_{2}$ is even, and that $b_{1}$ and $b_{2}$ are odd. We write $b_{1}=4 k+r, b_{2}=4 j+s$, with $r, s=+1$ or +3 . Then

$$
b_{1}-b_{2}=4(k-j)+(r-s)=K\left(d_{1}-d_{2}\right) \equiv 0 \quad(\bmod 4) .
$$

Hence $r=s$, and $b^{\prime \prime}=2(k+j)+r$, which is odd. Thus $n=1$.

We now compound the first and second forms, defining $b^{\prime \prime}, m$, and $n$ as above, and solving $d_{1} x+\left(b_{1}+b_{2}\right) y / 2=m$ for $x$ and $y$. Then

$$
\begin{aligned}
m z & \equiv\left(b^{\prime \prime}-b_{1}\right) x-d_{1}^{2} y \quad\left(\bmod d_{2}\right) \\
& \equiv\left(b_{2}-b_{1}\right) x / 2-d_{1}^{2} y \equiv(-K / 2) x\left(d_{1}-d_{2}\right)-d_{1}^{2} y \\
& \equiv(-K / 2) x d_{1}-d_{1}^{2} y \equiv(-K / 2)\left(m-y\left(b_{1}+b_{2}\right) / 2\right)-d_{1}^{2} y \\
& \equiv(-K m / 2)+y K\left(b_{1}+b_{2}\right) / 4-d_{1}^{2} y .
\end{aligned}
$$

But $K\left(b_{1}+b_{2}\right) / 4=d_{1}^{2}+d_{1} d_{2}+d_{2}^{2} \equiv d_{1}^{2}\left(\bmod d_{2}\right)$, hence

$$
\begin{aligned}
m z & \equiv-K m / 2 \quad\left(\bmod d_{2}\right), \\
z & \equiv-K / 2 \quad\left(\bmod d_{2}\right), \quad \text { since }\left(m, d_{2}\right)=1 .
\end{aligned}
$$

The compounded form is thus $\left(d_{1} d_{2}, b_{1}-K d_{1}, C\right)$, for some integer $C$. From Eqs. (6), we see that $C=d_{3}$, so the composition is, remembering $b_{1}-K d_{1}=$ $b_{2}-K d_{2}=b_{3}-K d_{3}=L$,

$$
\left(d_{1} d_{2}, b_{3}-K d_{3}, d_{3}\right) \sim\left(d_{3},-b_{3}, d_{3}^{2}\right) \sim\left(d_{3}, b_{3}, d_{3}^{2}\right)^{-1} \text {. }
$$

(ii) $K$ is odd, and $D \equiv-1(\bmod 8)$. From (7), $4 \mid\left(b_{1}+b_{2}\right)$, hence $b_{1} \equiv-b_{2}$ $(\bmod 4)$. Write $b_{1}=4 k+r$, and $b_{2}=4 j+r+2$. Then $K=(4(k-j)-2) /\left(d_{1}-d_{2}\right)$, which implies that exactly one of $d_{1} / 2$ and $d_{2} / 2$ is odd. We assume, without loss of generality, that $d_{1} / 2$ is odd. Then $m=n=2$. We solve for $x, y$, and $z$ as before, and find that $z \equiv-K\left(\bmod d_{2} / 2\right)$. The compounded form is then $\left(d_{1} d_{2} / 4, b_{1}-K d_{1}, t\right)$. where we again use (6) to find the third term of the form. Since $t \equiv 1(\bmod 8)$, and $(u-K t) / 4=L$, which is odd, we see that $(K-u) / 4$ is odd. This implies that

$$
(u-K t) / 4 \equiv(1-t) u / 4+t \quad(\bmod 2 t)
$$

hence 


$$
F_{1} \circ F_{2} \sim\left(d_{1} d_{2} / 4, L, t\right) \sim\left(t,-(1-t) u / 4+t, v_{1}\right) \sim F_{3}^{-1} .
$$

(iii) $K$ is odd, and $D \equiv-5(\bmod 8)$. Since $d_{1}$ is odd, $m=n=1$. Again, we solve for $x, y$, and $z$, and find this time that $z \equiv\left(d_{2}-K\right) / 2\left(\bmod d_{2}\right)$. Then $F_{1}$ 。 $F_{2} \sim\left(d_{1} d_{2}, L+d_{1} d_{2}, J\right)$, where $J=\left(\left(L+d_{1} d_{2}\right)^{2}+D\right) / 4 d_{1} d_{2}$. Using (6), we find $-L-d_{1} d_{2}+4 J=L+t$, so $\left(d_{1} d_{2}, L+d_{1} d_{2}, J\right) \sim(J, L+t, t)$. Now, $(u-K t) / 4$ $=L$ is even, and $t \equiv 1(\bmod 8)$; we see $K \equiv u(\bmod 8)$. This is sufficient to prove that

$$
L+t \equiv u(1-t) / 4+t \quad(\bmod 2 t)
$$

hence

$$
(J, L+t, t) \sim\left(t,-(1-t) u / 4+t, v_{1}\right) \sim F_{3}^{-1},
$$

and the theorem is proved.

3. The odd discriminants of complex quadratic number fields are congruent either to -1 or -5 modulo 8 . We have so far applied the method of Section 1 only to the former type, beginning with -3321607 as the constant term. The odd discriminants thus obtained are always congruent to -1 modulo 8 because the $a$ of Eq. (1a) are always even. One could also begin with the constant term $-3640387 \equiv-5(\bmod 8)$, but the series which arise are inherently less useful, half of the discriminants being even and not fundamental. (It follows from (6) and the representation of $2 K$ that the $c_{i}$ are even if $D \equiv 1(\bmod 8)$ and that exactly two of the $c_{i}$ are odd if $D \equiv 5(\bmod 8)$. Thus, for $D \equiv 5(\bmod 8)$, the $a_{i}$ are even, if they are integral, hence $S_{i}(x)$ is even for odd integers $x$.)

Using Scholz's theorem [8], we can deduce the 3-rank of the real fields $Q(\sqrt{3 D})$ from that of the complex fields $Q(\sqrt{-D})$. However, in all of the eight discriminants found the 3-rank of the corresponding real field is, by the theorem, only two.

The major question which we have not been able to answer has already been raised: Why should one 3-cycle of solutions to $2 K=u^{2}+3 v^{2}$ yield integer coefficients $a_{i}$, and one 3-cycle not do so? We have noted further that when four 3-cycles exist, as for $K=728$, only one of these yields integer values $a_{i}$.

The fact that quartic polynomials represent discriminants of complex quadratic fields only finitely many times is a limiting condition on the method we have developed. We considered cubic and sextic polynomials first, as they do not have this property. If, in (2), one lets the $S_{i}$ and $C_{i}$ be linear, one finds easily that there are no solutions with rational integer coefficients. We were successful in solving (2) with the $S_{i}$ and $C_{i}$ monic cubic and quadratic polynomials, respectively, so that $\Delta(x)$ has leading term $-3 x^{6}$. However, $\Delta(x)$ was inherently not squarefree except in rare instances. For this reason we concentrated our attention on the quartic polynomials of Section 1.

Appendix A. The basic method of computation of the class numbers and class groups was suggested by Atkin, who used it to compute some tables of his own. The method of computing the class group was published by Shanks in [9]. The program was written entirely in FORTRAN, and was run in segments, each segment computing the class numbers, groups, and statistics for a block of discriminants of length 200000 , 
even and odd discriminants comprising different segments. (Thus, one such segment contained the odd discriminants $-D, 600000<D<800000$.) An array of length 50000, CLANO, was used to store the needed information on the discriminants, CLANO $(N)$ corresponding to $D=D(N)=4 N+i+S$, where $S$ is the appropriate multiple of 200000 for the program segment being computed, and $i$ is 0 or -1 , depending on the parity of the discriminants in that segment.

The discriminants were first factorized, adding 10000 to CLANO for each prime factor, and adding - 1000000 if a factor appeared twice. This allowed an easy separation of the fundamental from the nonfundamental discriminants, as neither the number of factors nor the class number would be large enough to make the entry of CLANO positive later. Also, since the actual class number is only about $\sqrt{D}$, the number of genera could be computed from the number of factors of $D$ by using FORTRAN arithmetic to separate the digits of CLANO from one another.

The class numbers for all discriminants in a segment were computed together by executing a triple loop on the coefficients $b, a$, and $c$ (from the outermost to the innermost loop) of the binary quadratic form $(a, b, c)$ and then incrementing the counter $\operatorname{CLANO}(N)$ for the appropriate $-D(N)=b^{2}-4 a c$. That is, instead of fixing a value for $D$ and then computing the reduced forms for that $D$, we computed all reduced forms with discriminants in the range of the segment and kept a count for each $D$. Some care was taken to optimize these loops and remove all unnecessary multiplication; $D$ was computed each time by adding to the previous $D$, rather than by direct computation.

The primary list of discriminants, number of genera, and number of forms per genus was now computed and printed, and a secondary list of "possibly noncyclic" groups was extracted. (In what follows, we describe the computation for an odd $p$-Sylow subgroup; the suitable changes for the case $p=2$ are easy, but the description in words is cumbersome.) A group was "possibly noncyclic" in the $p$-Sylow subgroup if $p^{2} \mid h$, where $h$ is the order of the group (the class number). Each group in the list of possibles was then tested in the following manner: If $h=p^{i} m,(m, p)=1$, one chooses "at random" (to be described in the next paragraph) up to eleven forms of the group and computes the $h p^{1-i}$ th power of each. Should any of these not be the identity, the group is cyclic, and we proceed to the next discriminant. If that power of each of the eleven forms in the identity, the group is assumed to be noncyclic, and an actual computation of the $p$-Sylow subgroup is made, under the assumption that the group is noncyclic.

We now describe the "random" method for obtaining forms of a given discriminant: If, for a given $-D$, there is an $a$ such that $-D$ is a quadratic residue modulo $a$, then there exists a form of discriminant $-D$ and leading coefficient $a$. We checked through the odd primes under 1000 , in increasing order, to find one for which $-D$ was a residue. Taking this prime for the coefficient $a$, we searched for the smallest $b$ such that $b^{2} \equiv-D(\bmod a)$, obtaining a form $(a, b, c)$, which we then reduced. A reasonably thorough testing of this method and a continued use of it have not revealed any obvious patterns in the forms produced, so we have assumed that it was sufficiently random for our purposes. 
Appendix B. The smallest $D$ for which the class group of $Q(\sqrt{-D})$ is noncyclic in the $p$-Sylow subgroup are listed in Table 1, even and odd discriminants being listed where known. No even discriminants with a noncyclic 17-Sylow subgroup were found. We note also that $D=1016083,1438483,1663747,2407267$, and 2942227 , all (necessarily) prime, have class group exactly $C(13) \times C(13)$.

Of interest also are the groups which are noncyclic in more than one Sylow subgroup. There were 418 of these in all. Most of these were $C(12) \times C(12)$, the first (even and odd $D$ ) being 64952 and 104255 . The first $C(20) \times C(20)$ subgroups occurred for $D=472196$ and 280847 ; the first $C(28) \times C(28)$ subgroups were for $D=$ 858296 and 465719 . The class group $C(15) \times C(15)$ for $D=119191$ had been found some time ago by A. O. L. Atkin; surprisingly, the next examples are quite large. We list them in Table 2. Throughout Tables $1-10$, column $\mathrm{A}$ is the discriminant $-D$, column B the factorization of $D$, and column $\mathrm{C}$ the class group of $Q(\sqrt{-D})$, where, for example, $15 \times 15$ signifies the group $C(15) \times C(15)$.

The question of which finite Abelian groups occur as class groups of quadratic fields has been discussed at length in the literature (for example, [15], [16], and [17]). In Tables 11-13 we list some of the more unusual groups that occurred. Columns A through $D$ are, respectively, the group, the smallest $D$ for which that group occurred, the factorization of $D$, and the number of occurrences of that group. In Table 11 are listed, for $p \geqslant 3$, the groups which are themselves, or whose principal genera are, $p$-groups $C\left(p^{a}\right) \times C\left(p^{b}\right)$ with $a$ and $b \geqslant 2$. Note the single example of $p \geqslant 5$. In Table 12 are the 2-groups which contain a $C(4) \times C(4) \times C(4)$ subgroup, that is, the 2-groups whose principal genus has rank 3 . There were no groups found with a subgroup $C\left(p^{a}\right) \times C\left(p^{b}\right), a$ and $b \geqslant 3$ and $p>2$; we list in Table 13, however, the class groups whose principal genus contained a subgroup $C\left(2^{a}\right) \times C\left(2^{b}\right), a$ and $b \geqslant 3$.

Finally, in Tables 14 and 15 we collect some statistics on the frequency of occurrence of noncyclic groups.

\section{TABLE 1}

\begin{tabular}{rrl}
$\mathrm{P}$ & \multicolumn{1}{|c}{} & \multicolumn{1}{c}{ B } \\
3 & -3299 & prime \\
5 & -11199 & 3.3733 \\
7 & -63499 & prime \\
11 & -65591 & 107.613 \\
13 & $-228679 *$ & 11.20789 \\
17 & $-1997799 *$ & 3.59 .11287 \\
19 & $-373391 *$ & 67.5573
\end{tabular}

\begin{tabular}{r|clr}
\multicolumn{1}{c}{$\mathrm{C}$} & \multicolumn{1}{c}{$\mathrm{A}$} & \multicolumn{1}{c}{$\mathrm{B}$} & \multicolumn{1}{c}{$\mathrm{C}$} \\
$3 \times 9$ & -3896 & 8.487 & $3 \times 12$ \\
$5 \times 20$ & -17944 & 8.2243 & $5 \times 10$ \\
$7 \times 7$ & -159592 & 8.19949 & $7 \times 14$ \\
$11 \times 22$ & $-580424^{*}$ & 8.13 .5581 & $22 \times 22$ \\
$13 \times 26$ & $-703636^{*}$ & 4.175909 & $13 \times 26$ \\
$34 \times 34$ & $-3419828^{*}$ & 4.854957 & $19 \times 38$
\end{tabular}

TABLE 2

\begin{tabular}{cll|lll}
$\underline{A}$ & \multicolumn{1}{c}{$\underline{\mathrm{B}}$} & $\underline{\mathrm{C}}$ & \multicolumn{1}{c}{$\underline{\mathrm{A}}$} & $\underline{\mathrm{B}}$ & $\underline{\mathrm{C}}$ \\
-119191 & prime & $15 \times 15$ & -3150391 & prime & $15 \times 105$ \\
-2075343 & 3.17 .40693 & $30 \times 30$ & -3358427 & 349.9623 & $15 \times 30$ \\
-2403659 & prime & $15 \times 45$ & -3492051 & 3.941 .1237 & $30 \times 30$ \\
-2690455 & 5.37 .14543 & $30 \times 30$ & -3561799 & prime & $21 \times 63$ \\
-2766392 & 8.59 .5861 & $30 \times 30$ & -3860484 & 4.3 .321707 & $30 \times 30$ \\
-2982783 & 3.809 .1229 & $30 \times 30$ & -3862148 & 4.67 .14411 & $30 \times 30$ \\
-3072743 & 83.37021 & $15 \times 60$ & -3874699 & 467.8297 & $15 \times 30$
\end{tabular}


TABLE 3

Groups with a $20 \times 20$ subgroup

\begin{tabular}{cll|lll}
\multicolumn{1}{c}{$\underline{\mathrm{A}}$} & \multicolumn{1}{c}{$\underline{\mathrm{B}}$} & \multicolumn{1}{c}{$\underline{\mathrm{C}}$} & $\underline{\mathrm{A}}$ & \multicolumn{1}{c}{$\underline{\mathrm{B}}$} & $\underline{\mathrm{C}}$ \\
-280847 & 7.53 .757 & $20 \times 20$ & -2540344 & $\mathbf{8 . 1 7 . 1 8 6 7 9}$ & $20 \times 20$ \\
-458695 & 5.199 .461 & $20 \times 20$ & -2664955 & 5.29 .18379 & $20 \times 20$ \\
-472196 & 4.97 .1217 & $20 \times 20$ & -2713144 & 8.7 .48449 & $20 \times 20$ \\
-1323896 & 8.7 .47 .503 & $2 \times 20 \times 20$ & -2729255 & 5.19 .28729 & $20 \times 60$ \\
-1567495 & 5.251 .1249 & $20 \times 40$ & -2814299 & 17.19 .8713 & $20 \times 40$ \\
-1627451 & 7.29 .8017 & $20 \times 20$ & -2910615 & 3.5 .61 .3181 & $2 \times 20 \times 20$ \\
-1633487 & 19.149 .577 & $20 \times 40$ & -2922488 & 8.401 .911 & $20 \times 40$ \\
-1663023 & 3.457 .1213 & $20 \times 40$ & -2925944 & 8.7 .52249 & $20 \times 40$ \\
-1751335 & 5.23 .97 .157 & $2 \times 20 \times 20$ & -2985995 & 5.199 .3001 & $20 \times 40$ \\
-1847795 & 5.101 .3659 & $20 \times 20$ & -3044455 & 5.41 .14851 & $20 \times 40$ \\
-2007172 & 4.337 .1489 & $20 \times 20$ & -3174951 & 3.13 .81409 & $20 \times 80$ \\
-2025560 & 8.5 .79 .641 & $2 \times 20 \times 20$ & -3256568 & 8.7 .58153 & $20 \times 40$ \\
-2176955 & 5.11 .39581 & $20 \times 20$ & -3270307 & 17.47 .4093 & $20 \times 20$ \\
-2295736 & 8.31 .9257 & $20 \times 20$ & -3390483 & 3.457 .2473 & $20 \times 20$ \\
-2326904 & 8.239 .1217 & $20 \times 60$ & -3401615 & 5.7 .17 .5717 & $2 \times 20 \times 40$ \\
-2452439 & 11.113 .1973 & $20 \times 120$ & -3642743 & 13.17 .53 .311 & $2 \times 20 \times 40$ \\
-2524247 & 11.29 .41 .193 & $2 \times 20 \times 40$ & -3972344 & 8.97 .5119 & $20 \times 80$
\end{tabular}

TABLE 4

Groups with a $28 \times 28$ subgroup

A

$-465719$

$-632687$

$-858296$

$-2471624$

\begin{tabular}{lc}
\multicolumn{1}{c}{$\underline{\text { B }}$} & $\underline{\text { C }}$ \\
37.41 .307 & $28 \times 28$ \\
11.113 .509 & $28 \times 28$ \\
8.17 .6311 & $28 \times 28$ \\
8.521 .593 & $28 \times 28$
\end{tabular}

A

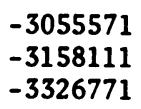

$-3326771$
$\begin{array}{cc}\underline{B} & \underline{C} \\ 37.269 .307 & 28 \times 28 \\ 11.53 .5417 & 28 \times 84 \\ 7.137 .3469 & 28 \times 28\end{array}$

TABLE 5

Noncyclic 5-Sylow subgroups for $D<100000$

$\underline{A}$
-11199
-12451
-17944
-30263
-33531
-37363
-38047
-39947
-42871
-50783
-53079
-54211
-58424
-61556
-62632
-63411
-64103
-65784
-66328
-67031 B

3.3733

prime

8.2243

53.571

3.11177

prime

prime

43. 929

43.997

43. 1181

3. 13.1361

23. 2357

8.67.109

4.11. 1399

8.7829

3. 23.919

13.4931

8. 3.2741

8. 8291

17.3943

\begin{tabular}{|c|c|}
\hline$\underline{\mathrm{C}}$ & $\underline{\mathbf{A}}$ \\
\hline $5 \times 20$ & -67063 \\
\hline $5 \times 5$ & -67128 \\
\hline $5 \times 10$ & -69811 \\
\hline $5 \times 30$ & -72084 \\
\hline $5 \times 10$ & -74051 \\
\hline $5 \times 5$ & -75688 \\
\hline $5 \times 15$ & -81287 \\
\hline $5 \times 10$ & -83767 \\
\hline $5 \times 30$ & -84271 \\
\hline $5 \times 50$ & -85099 \\
\hline $10 \times 20$ & -85279 \\
\hline $5 \times 10$ & -87971 \\
\hline $10 \times 10$ & -89751 \\
\hline $10 \times 20$ & -90795 \\
\hline $5 \times 10$ & -90868 \\
\hline $10 \times 10$ & -92263 \\
\hline $5 \times 40$ & -98591 \\
\hline $10 \times 10$ & -99031 \\
\hline $5 \times 10$ & -99743 \\
\hline & \\
\hline
\end{tabular}

C

$5 \times 30$

$10 \times 10$

$5 \times 10$

$10 \times 10$

$5 \times 35$

$5 \times 10$

$5 \times 50$

$5 \times 30$

$10 \times 20$

$5 \times 10$

$5 \times 40$

$10 \times 10$

$5 \times 60$

$10 \times 10$

$5 \times 10$

$5 \times 30$

$5 \times 90$

$5 \times 30$

$5 \times 60$ 
TABLE 6

Noncyclic 7-Sylow subgroups for $D<500000$

\begin{tabular}{rll|lll}
$\mathbf{A}$ & \multicolumn{1}{c}{$\underline{\mathrm{B}}$} & \multicolumn{1}{c}{$\underline{\mathrm{C}}$} & \multicolumn{1}{c}{ A } & \multicolumn{1}{c}{ B } & \multicolumn{1}{c}{$\underline{\mathrm{C}}$} \\
-63499 & prime & $7 \times 7$ & -268739 & 31.8669 & $7 \times 28$ \\
-118843 & prime & $7 \times 7$ & -272179 & prime & $7 \times 21$ \\
-124043 & 163.761 & $7 \times 14$ & -275636 & 4.68909 & $7 \times 42$ \\
-149519 & prime & $7 \times 91$ & -294935 & 5.61 .967 & $14 \times 28$ \\
-159592 & 8.19949 & $7 \times 14$ & -299627 & 103.2909 & $7 \times 28$ \\
-170679 & 3.56893 & $7 \times 56$ & -301211 & prime & $7 \times 21$ \\
-183619 & 139.1321 & $7 \times 14$ & -308531 & 29.10639 & $7 \times 28$ \\
-185723 & prime & $7 \times 21$ & -318547 & 113.2819 & $7 \times 14$ \\
-220503 & 3.31 .2371 & $14 \times 28$ & -346883 & 19.18257 & $7 \times 28$ \\
-226691 & prime & $7 \times 35$ & -361595 & 5.13 .5563 & $14 \times 14$ \\
-227387 & prime & $7 \times 21$ & -366295 & 5.73259 & $7 \times 56$ \\
-227860 & 4.5 .11393 & $14 \times 14$ & -373655 & 5.74731 & $7 \times 84$ \\
-236931 & 3.78977 & $7 \times 14$ & -465719 & 37.41 .307 & $28 \times 28$ \\
-240347 & prime & $7 \times 21$ & -480059 & prime & $7 \times 49$ \\
-240655 & 5.48131 & $7 \times 28$ & -489576 & 8.3 .20399 & $14 \times 28$ \\
-247252 & 4.61813 & $7 \times 14$ & -491767 & 37.13291 & $7 \times 42$ \\
-260111 & prime & $7 \times 77$ & & &
\end{tabular}

TABLE 7

Noncyclic 11-Sylow subgroups

\begin{tabular}{cll|lll}
$\underline{\mathrm{A}}$ & \multicolumn{1}{c}{$\underline{\mathrm{B}}$} & $\underline{\mathrm{C}}$ & $\underline{\mathrm{A}}$ & $\underline{\mathrm{B}}$ & $\underline{\mathrm{C}}$ \\
-65591 & 107.613 & $11 \times 22$ & -2659099 & 23.115613 & $11 \times 22$ \\
-126407 & 19.6653 & $11 \times 22$ & -2661639 & 3.17 .52189 & $22 \times 44$ \\
-175031 & 383.457 & $11 \times 66$ & -2668715 & 5.7 .76249 & $22 \times 22$ \\
-272231 & prime & $11 \times 33$ & -2697779 & 7.385397 & $11 \times 66$ \\
-423335 & 5.11 .43 .179 & $2 \times 22 \times 22$ & -2741799 & 3.913933 & $11 \times 88$ \\
-527019 & 3.175673 & $11 \times 22$ & -2747743 & 43.63901 & $11 \times 66$ \\
-580424 & 8.13 .5581 & $22 \times 22$ & -2828680 & 8.5 .70717 & $22 \times 22$ \\
-593183 & prime & $11 \times 77$ & -2913679 & 109.26731 & $11 \times 132$ \\
-680767 & prime & $11 \times 33$ & -2934312 & 8.3 .122263 & $22 \times 22$ \\
-694907 & 571.1217 & $11 \times 22$ & -2946299 & prime & $11 \times 55$ \\
-767147 & prime & $11 \times 33$ & -3032179 & prime & $11 \times 33$ \\
-857099 & prime & $11 \times 33$ & -3037459 & 127.23917 & $11 \times 44$ \\
-1161239 & prime & $11 \times 99$ & -3130027 & prime & $11 \times 33$ \\
-1314676 & 4.11 .29879 & $22 \times 22$ & -3131864 & 8.23 .17021 & $22 \times 44$ \\
-1451639 & 7.207377 & $11 \times 132$ & -3152315 & 5.103 .6121 & $22 \times 22$ \\
-1471423 & prime & $11 \times 33$ & -3251123 & 113.28771 & $11 \times 44$ \\
-1654147 & 11.150377 & $11 \times 22$ & -3301883 & 13.499 .509 & $22 \times 22$ \\
-1689371 & 509.3319 & $11 \times 66$ & -3418507 & 149.22943 & $11 \times 22$ \\
-1734395 & 5.13 .26683 & $22 \times 22$ & -3426456 & 8.3 .11 .12979 & $2 \times 22 \times 22$ \\
-1764687 & 3.588229 & $11 \times 44$ & -3431179 & prime & $11 \times 33$ \\
-1963419 & 3.167 .3919 & $22 \times 22$ & -3497892 & 4.3 .291491 & $22 \times 22$ \\
-2148079 & 307.6997 & $11 \times 110$ & -3645907 & 883.4129 & $11 \times 22$ \\
-2253971 & prime & $11 \times 55$ & -3781607 & 173.21859 & $11 \times 110$ \\
-2608212 & 4.3 .217351 & $22 \times 22$ & -3810631 & 11.346421 & $11 \times 132$ \\
-2628123 & 3.876041 & $11 \times 22$ & -3894239 & prime & $11 \times 297$
\end{tabular}


TABLE 8

Noncyclic 13-Sylow subgroups

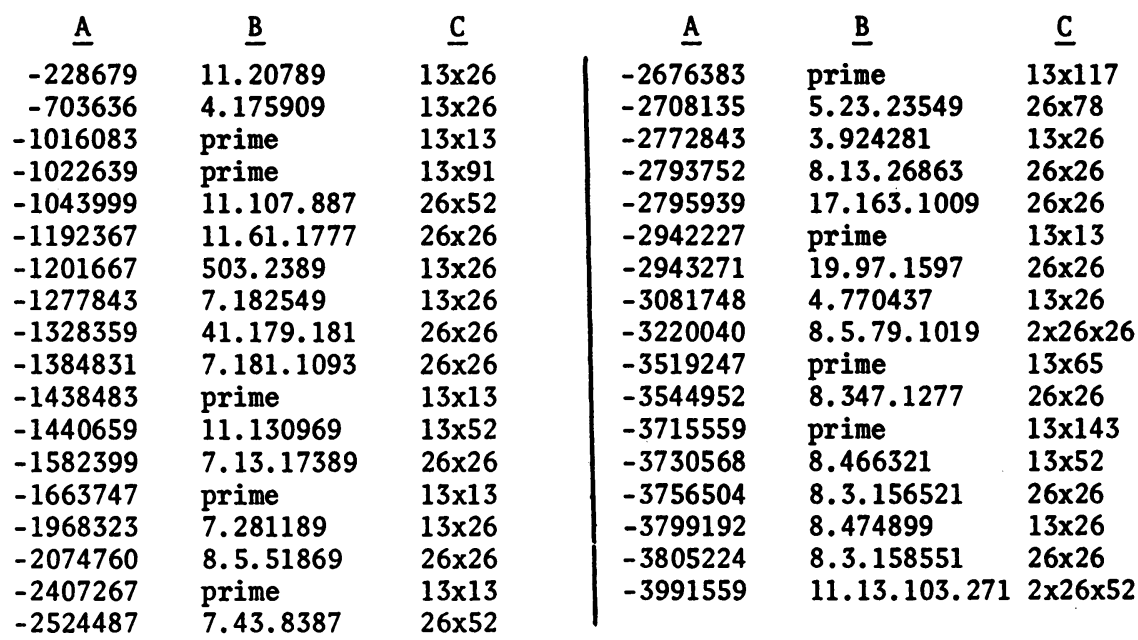

TABLE 9

Noncyclic 17-Sylow subgroups

A

$-1997799$

$-2667895$

$-2890903$
B

$5.17 .31387 \quad 34 \times 34$ $1019.2837 \quad 17 \times 34$

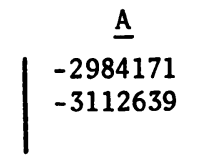

B

$\underline{\mathrm{C}}$

47.63493

prime

$17 \times 34$

$17 \times 85$

TABLE 10

Noncyclic 19-Sylow subgroups

\begin{tabular}{clllll}
$\underline{\mathrm{A}}$ & \multicolumn{1}{c}{$\underline{\mathrm{B}}$} & $\underline{\mathrm{C}}$ & $\underline{\mathrm{A}}$ & $\underline{\mathrm{B}}$ & $\underline{\mathrm{C}}$ \\
-373391 & 67.5573 & $19 \times 38$ & -3419828 & 4.854957 & $19 \times 38$ \\
-1078919 & prime & $19 \times 57$ & -3479127 & 3.1159709 & $19 \times 38$ \\
-2505135 & 3.5 .167009 & $38 \times 38$ & -3837956 & 4.959489 & $19 \times 76$
\end{tabular}

TABLE 11

\begin{tabular}{lrlllllr}
$\underline{A}$ & $\underline{B}$ & \multicolumn{1}{c}{$\underline{\mathrm{C}}$} & $\underline{\mathrm{D}}$ & \multicolumn{1}{c}{ A } & $\underline{\mathrm{B}}$ & $\underline{\mathrm{C}}$ & $\underline{\mathrm{D}}$ \\
$9 \times 9$ & -134059 & prime & 3 & $18 \times 18$ & -727087 & 37.43 .457 & 18 \\
$9 \times 27$ & -351751 & prime & 4 & $18 \times 54$ & -1871295 & 3.5 .124753 & 7 \\
$9 \times 81$ & -1332167 & prime & 1 & $2 \times 18 \times 18$ & -2442020 & 4.5 .7 .17443 & 5 \\
$9 \times 18$ & -208084 & 4.52021 & 5 & $25 \times 50$ & -1390367 & 11.126397 & 1 \\
$9 \times 54$ & -690503 & 11.62773 & 9 & & & &
\end{tabular}

TABLE 12

\begin{tabular}{lcllllll}
\multicolumn{1}{c}{ A } & $\underline{B}$ & \multicolumn{1}{c}{ C } & $\underline{D}$ & \multicolumn{1}{c}{ A } & \multicolumn{1}{c}{ B } & \multicolumn{1}{c}{ C } & D \\
$4 \times 4 \times 8$ & -503659 & 13.17 .43 .53 & 4 & $4 \times 8 \times 8$ & -2209467 & 3.13 .181 .313 & 4 \\
$4 \times 4 \times 16$ & -550712 & 8.23 .41 .73 & 6 & $4 \times 8 \times 16$ & -1456131 & 3.61 .73 .109 & 1 \\
$4 \times 4 \times 32$ & -863455 & 5.19 .61 .149 & 5 & $2 \times 4 \times 4 \times 16$ & -2172651 & 3.13 .17 .29 .113 & 2 \\
$4 \times 4 \times 64$ & -3600632 & 8.7 .113 .569 & 1 & $2 \times 2 \times 4 \times 4 \times 8$ & -2188920 & 8.3 .5 .17 .29 .37 & 1
\end{tabular}


TABLE 13

\begin{tabular}{lrlr|lllr}
\multicolumn{1}{c}{ A } & \multicolumn{1}{c}{ B } & \multicolumn{1}{c}{ C } & $\underline{\text { D }}$ & \multicolumn{1}{c}{ A } & \multicolumn{1}{c}{ B } & \multicolumn{1}{c}{ C } & $\underline{\text { D }}$ \\
$16 \times 16$ & -618947 & 7.29 .3049 & 16 & $16 \times 128$ & -3194495 & 5.29 .22031 & 1 \\
$16 \times 48$ & -936183 & 3.313 .997 & 12 & $32 \times 32$ & -236559 & 3.421 .1873 & 1 \\
$16 \times 80$ & -1831031 & 29.103 .613 & 3 & $2 \times 16 \times 16$ & -804639 & 3.11 .37 .659 & 17 \\
$16 \times 112$ & -1602095 & 5.11 .29129 & 1 & $2 \times 16 \times 48$ & -3228215 & 5.17 .163 .233 & 2 \\
$16 \times 32$ & -971095 & 5.359 .541 & 17 & $2 \times 16 \times 32$ & -1987215 & 3.5 .17 .7793 & 2 \\
$16 \times 96$ & -2747399 & 43.181 .353 & 1 & $2 \times 2 \times 16 \times 16$ & -3909576 & 8.3 .11 .59 .251 & 1 \\
$16 \times 64$ & -1008095 & 5.11 .18329 & 6 & & & &
\end{tabular}

TABLE 14

$\begin{array}{lrccccc} & \underline{A} & \underline{B} & \underline{C} & \underline{D} & \underline{E} & \underline{\mathbf{F}} \\ \text { Odd D } & 810578 & 265739 & 32.78 & 32507 & 12.23 & 4.01 \\ \text { Even D } & 405276 & 166296 & 41.03 & 19345 & 11.63 & 4.77 \\ \text { Total } & 1215854 & 432035 & 35.53 & 51852 & 12.00 & 4.26\end{array}$

$A=$ number of fundamental discriminants

$B=$ number of possibly noncyclic groups

$\mathrm{C}=100 \times \mathrm{B} / \mathrm{A}$

Denumber of actually noncyclic slass groups

$E=100 \times D / B$

$\mathrm{F}=100 \times \mathrm{D} / \mathrm{A}$

TABLE 15

Noncyclic p-Sylow subgroups

\begin{tabular}{|c|c|c|c|c|c|c|}
\hline $\mathrm{p}=2$ & $\underline{\mathbf{A}}$ & $\underline{B}$ & $\underline{\mathrm{C}}$ & $\underline{\mathrm{D}}$ & $\underline{E}$ & $\underline{\mathbf{F}}$ \\
\hline $\begin{array}{l}\text { Odd D } \\
\text { Even D } \\
\text { Total }\end{array}$ & $\begin{array}{r}810578 \\
405276 \\
1215854\end{array}$ & $\begin{array}{l}122971 \\
101029 \\
224000\end{array}$ & $\begin{array}{l}15.71 \\
24.93 \\
18.42\end{array}$ & $\begin{array}{l}20837 \\
13862 \\
34699\end{array}$ & $\begin{array}{l}16.94 \\
13.72 \\
15.49\end{array}$ & $\begin{array}{l}2.57 \\
3.42 \\
2.85\end{array}$ \\
\hline $\mathrm{p}=3$ & $\underline{\mathbf{A}}$ & $\underline{B}$ & $\underline{\mathrm{C}}$ & $\underline{\mathrm{D}}$ & $\underline{E}$ & $\underline{F}$ \\
\hline $\begin{array}{l}\text { Odd D } \\
\text { Even D } \\
\text { Total }\end{array}$ & $\begin{array}{r}810578 \\
405276 \\
1215854\end{array}$ & $\begin{array}{r}115904 \\
57264 \\
173168\end{array}$ & $\begin{array}{l}14.30 \\
14.13 \\
14.24\end{array}$ & $\begin{array}{r}10132 \\
4832 \\
14964\end{array}$ & $\begin{array}{l}8.74 \\
8.44 \\
8.64\end{array}$ & $\begin{array}{l}1.25 \\
1.19 \\
1.23\end{array}$ \\
\hline $\mathrm{p}=5$ & $\underline{\mathbf{A}}$ & $\underline{B}$ & $\underline{\mathrm{C}}$ & $\underline{D}$ & $\underline{E}$ & $\underline{\mathbf{F}}$ \\
\hline $\begin{array}{l}\text { Odd D } \\
\text { Even D } \\
\text { Tota1 }\end{array}$ & $\begin{array}{r}810578 \\
405276 \\
1215854\end{array}$ & $\begin{array}{l}37485 \\
17639 \\
55124\end{array}$ & $\begin{array}{l}4.62 \\
4.35 \\
4.53\end{array}$ & $\begin{array}{r}1419 \\
648 \\
2067\end{array}$ & $\begin{array}{l}3.79 \\
3.67 \\
3.75\end{array}$ & $\begin{array}{l}.18 \\
.16 \\
.17\end{array}$ \\
\hline $\mathrm{p}=7$ & $\underline{\mathbf{A}}$ & $\underline{B}$ & $\underline{\mathrm{C}}$ & $\underline{\mathrm{D}}$ & $\underline{E}$ & $\underline{F}$ \\
\hline $\begin{array}{l}\text { Odd D } \\
\text { Even D } \\
\text { Total }\end{array}$ & $\begin{array}{r}810578 \\
405276 \\
1215854\end{array}$ & $\begin{array}{r}16877 \\
7620 \\
24497\end{array}$ & $\begin{array}{l}2.08 \\
1.88 \\
2.01\end{array}$ & $\begin{array}{l}295 \\
149 \\
444\end{array}$ & $\begin{array}{l}1.75 \\
1.96 \\
1.81\end{array}$ & $\begin{array}{l}.04 \\
.04 \\
.04\end{array}$ \\
\hline
\end{tabular}

$A=$ number of fundamental discriminants

$B=$ number of possibly noncyclic $p$-Sylow subgroups $\mathrm{C}=100 \times \mathrm{B} / \mathrm{A}$

$D=$ number of actually noncyclic p-Sylow subgroups

$E=100 \times D / B$

$\mathrm{F}=100 \times \mathrm{D} / \mathrm{A}$ 
1. MAURICE CRAIG, "A type of class group for imaginary quadratic fields," Acta Arith., v. 22, 1973, pp. 449-459. MR 47 \#6647.

2. DANIEL SHANKS \& PETER WEINBERGER, "A quadratic field of prime discriminant requiring three generators for its class group, and related theory," Acta Arith., v. 21, 1972, pp. 7187. MR 46 \#9003.

3. DANIEL SHANKS, "New types of quadratic fields having three invariants divisible by three," J. Number Theory, v. 4, 1972, pp. 537-556. MR 47 \#1775.

4. DANIEL SHANKS \& RICHARD SERAFIN, "Quadratic fields with four invariants divisible by three," Math. Comp., v. 27, 1973, pp. 183-187; Corrigendum, ibid., p. 1012. MR 48 \#8436a, b.

5. CAROL NEILD \& DANIEL SHANKS, "On the 3-rank of quadratic fields and the Eut. product," Math. Comp., v. 28, 1974, pp. 279-291.

6. F. DIAZ Y DIAZ, "Sur les corps quadratiques imaginaires dont le 3-rang du groupe des classes est supérieur à 1," Séminaire Delange-Pisot-Poitou, 1973/74, no. G15.

6a. DANIEL SHANKS, "Class groups of the quadratic fields found by Diaz y Diaz," Math. Comp., v. 30, 1976, pp. 173-178.

7. ERICH HECKE, Vorlesungen über die Theorie der algebraischen Zahlen, Chelsea, New York, 1970. MR 50 \#4524.

8. ARNOLD SCHOLZ, "Über die Beziehung der Klassenzahlen quadratischer Körper zueinander," J. Reine Angew. Math., v. 166, 1932, pp. 201-203.

9. DANIEL SHANKS, "Class number, a theory of factorization, and genera," Proc. Sympos. Pure Math., v. 20, Amer. Math. Soc., Providence, R. I., 1971, pp. 415-440. MR 47 \#4932.

10. R. A. LIPPMAN, "Note on irregular discriminants," J. London Math. Soc., v. 38, 1963, pp. 385-386. MR 28 \#1174.

11. E. T. ORDMAN, "Tables of class numbers for negative prime discriminants," UMT 29, Math. Comp., v. 23, 1969, p. 458.

12. M. NEWMAN, "Table of the class number $h(-p)$ for $p$ prime, $p \equiv 3(\bmod 4), 101987$ $<p<166807$," UMT 50, Math. Comp., v. 23, 1969, p. 683.

13. RICHARD B. LAKEIN \& SIGEKATU KURODA, "Tables of class numbers $h(-p)$ for fields $Q(\sqrt{-p}), p<465071$," UMT 39, Math. Comp., v. 24, 1970, pp. 491-493.

14. H. WADA, “A table of ideal class groups of imaginary quadratic fields," Proc. Japan Acad., v. 46, 1970, pp. 401-403.

15. S. CHOWLA, “An extension of Heilbronn's class number theorem," Quart. J. Math. Oxford Ser., v. 5, 1934, pp. 304-307.

16. W. NARKIEWICZ, "Class number and factorization in quadratic number fields," Colloq. Math., v. 17, 1967, pp. 167-190. MR 36 \#3750.

17. DANIEL SHANKS, “On Gauss's class-number problems,” Math Comp., v. 23, 1969, pp. 151-163. MR 41 \#6814. 